\section{POS1127 1 ULTRASOUND ASSESSMENT OF GOUT LESIONS IN AN ALGERIAN POPULATION WITH ASYMPTOMATIC HYPERURICEMIA}

B. Bengana ${ }^{1}$, A. Ladjouze-Rezig², S. Ayoub ${ }^{3}$, N. B. Raaf ${ }^{4}$, C. Aimeur ${ }^{5}$, A. Boukabous ${ }^{1}$, S. Lefkir ${ }^{1}{ }^{1}$ University Hospital of Beni Messous - Algiers, Rheumatology, BeniMessous, Algeria; ${ }^{2}$ Specialized hospital establishment of Benaknoune, Rheumatology, Benaknoune, Algeria; ${ }^{3}$ University hospital of Beni Messous, Internal Medicine, BeniMessous, Algeria; ${ }^{4}$ Hospital of Bitraria, Biochemistry, El Biar, Algeria; ${ }^{5}$ University hospital of Mustapha Bacha, Radiology, Sidi Mhamed, Algeria

Background: Hyperuricemia is a common biological abnormality, often clinically asymptomatic. However, it can announce a gout and be linked to many diseases such as metabolic syndrome, high blood pressure or kidney disease.

In fact, the majority of learned societies do not recommend any urate lowering therapy (ULT) as long as the hyperuricemia remains asymptomatic. But it turns out that part of the population with asymptomatic hyperuricemia (AH) develops a gout after a few years particularly with genetic predisposition, but also on certain risk factors that need to be confirmed.

By this way, musculoskeletal ultrasound (MUS) can detect "asymptomatic gout" by visualizing signs of urate deposits (UD) in subjects with $\mathrm{AH}$.

Objectives: Our main objective is already to estimate the prevalence of specific signs of gout in Algerian population with $\mathrm{AH}$ and assess the factors exposing to UD.

Methods: This is a descriptive cross-sectional study from January 2017 to February 2019, with the recruitment of subjects with $\mathrm{AH}$ and serum urate level > $60 \mathrm{mg} / \mathrm{L}$, who do not take any ULT and have not associated any chronic inflammatory rheumatism, where we performed a MUS of the knees, metatarsophalangeal joints MTP1, MTP2 and metacarpophalangeal joint MCP2 and MCP3 with the Achilles, patellar and quadricipital tendons.

Results: We retained 258 subjects with $\mathrm{AH}, 132$ women and 126 men (sex ratio $=0.95$ ), the mean age was 59 years, the mean body mass index (BMI) was $28.4 \mathrm{~kg} / \mathrm{m} 2,42$ patients were under diuretics, 37 patients reported being on low-dose of aspirin $100 \mathrm{mg}$ daily.

The mean rate of serum urate levels was $78 \pm 10 \mathrm{mg} / \mathrm{L}$, the prevalence of UD found at the MUS was 22\% $(n=58)$, among them $36 \%(21 / 58)$ had a sign of the double contour DC on the MTP1 and 29\% (17/58) on the knee, $7 \%(4 / 58)$ had tophi on the MTP1 and $3 \%(2 / 58)$ had urate aggregates. The factors reported to be linked to UD in the sample were: the male gender ( $p=$ $0.0016)$; the high uric acid level ( $p=0.0355)$; BMI $(p=0.0427)$; taking diuretics for women $(p=0.0002)$.

Conclusion: Through this work, it is clear that elementary ultrasound lesions related to gout disease are common in a population with $\mathrm{AH}$ and concerned one fifth of subjects in our study with a higher risk in men and subjects with obesity and high uric acid level, but also in women taking diuretics. These results need to be enhanced with a randomized controlled study in order to better determine the predisposing factors for gout in any subject with $\mathrm{AH}$.

Disclosure of Interests: None declared.

DOI: 10.1136/annrheumdis-2021-eular.1161

\begin{tabular}{|l|l}
\hline POS1128 & COMBINATION TREATMENT OF AR882, A NEW URAT1 \\
INHIBITOR, AND XANTHINE OXIDASE INHIBITORS \\
ALLOPURINOL OR FEBUXOSTAT: EFFECT ON URIC \\
ACID, HYPOXANTHINE AND XANTHINE IN PLASMA \\
OR SERUM AND URINE
\end{tabular}

Z. Shen ${ }^{1}$, C. Colton ${ }^{1}$, R. Yan ${ }^{1}$, E. Polvent ${ }^{1}$, V. Hingorani ${ }^{1}$, S. Yan ${ }^{1}$, L. T. Yeh ${ }^{1}$.

${ }^{1}$ Arthrosi Therapeutics Inc, Laguna Hills, California, United States of America

Background: Xanthine oxidase inhibitors (XOI) are commonly used as urate lowering therapy (ULT) for the treatment of gout. Allopurinol, the first-line treatment, demonstrates low response rate $(<40 \%)$, defined as serum urate (sUA) lowering effect below $6 \mathrm{mg} / \mathrm{dL}$, in multiple large-scale clinical trials. As recommended in EULAR guidelines and other literatures, targeting sUA $<5 \mathrm{mg} / \mathrm{dL}$ or even $<4 \mathrm{mg} / \mathrm{dL}$, provides a better opportunity to lower incidence of gout flare and resolution of tophi in gout patients. Febuxostat, a more potent XOI, has been classified as a second-line ULT agent due to increased cardiovascular risks in certain patient populations. For $\mathrm{XOI}$ intolerance and non-responders, replacing the agent with a potent URAT1 inhibitor or adding an URAT1 inhibitor onto a current treatment regimen provides opportunities to improve response rates in patients with refractory gout. AR882 is a uricosuric agent that blocks the reabsorption of uric acid in the apical side of renal tubule, hence increases excretion of uric acid into the urine. A phase 2a study has demonstrated the additive effects of AR882 in combination with allopurinol or febuxostat.

Objectives: To evaluate the effect of AR882 alone or in combination with allopurinol or febuxostat on circulating levels and urinary excretion of hypoxanthine, xanthine and uric acid. Furthermore, to elucidate the contribution of each drug towards the combination effect in SUA lowering.
Methods: Plasma, serum, and urine samples were collected from 17 patients with gout who received a once-daily dosing of AR882 $50 \mathrm{mg}$, allopurinol $300 \mathrm{mg}$ or febuxostat $40 \mathrm{mg}$, or in combination for one week in a phase 2a study. Samples were collected to measure hypoxanthine, xanthine and uric acid levels in plasma or serum and the amount of each excreted in the urine. Plasma $\mathrm{C}_{\text {max }}$ and AUC and 24-hour urine excretion amount $(\mathrm{mg})$ of hypoxanthine and xanthine were calculated by non-compartmental analysis method.

Results: In monotherapy, AR882 demonstrated better sUA lowering effect $(\downarrow 53 \%)$ compared to allopurinol $(\downarrow 35 \%)$ or febuxostat $(\downarrow 39 \%)$. Combination of AR882 and allopurinol lowered sUA by $66 \%$ while combination of AR882 and febuxostat lowered sUA by $71 \%$. Based on the change of xanthine in plasma following combination treatments, it can be calculated that allopurinol contributed $28 \%$ of the urate lowering effect, whereas AR882 contributed $38 \%$ of the effect. Similarly, febuxostat contributed $33 \%$ of the urate lowering effect and AR882 contributed to about $36-38 \%$ of the effect when used in combination. The combination treatments significantly increased the response ratio for patients achieving SUA levels $<5 \mathrm{mg} / \mathrm{dL}, 4 \mathrm{mg} / \mathrm{dL}$, and even $3 \mathrm{mg} / \mathrm{dL}$. In the combination with allopurinol, $100 \%, 100 \%, 100 \%$, and $44 \%$ patients achieved sUA $<6 \mathrm{mg} / \mathrm{dL},<5 \mathrm{mg} / \mathrm{dL}, 4 \mathrm{mg} /$ $\mathrm{dL}$, and $3 \mathrm{mg} / \mathrm{dL}$, respectively. Similar results were seen with the combination of AR882 and febuxostat. Treatment with allopurinol or febuxostat alone resulted in 8 to 10 -fold and 16 -fold increases of xanthine exposure, respectively. The combination of AR882 and allopurinol or febuxostat showed approximately 5-8 fold or 13-fold increases in plasma xanthine, respectively. Treatment with allopurinol or febuxostat alone resulted in 2-3 fold increase in hypoxanthine exposure. Relative changes of hypoxanthine were not significantly different in the presence versus absence of AR882. Increased excretion of xanthine and hypoxanthine was also observed in urine. AR882 was well tolerated in gout patients, showing a similar safety profile to that observed in healthy volunteer studies.

Conclusion: AR882 dose alone had no effect on plasma or urine hypoxanthine and xanthine levels. AR882 contributed to a greater portion of the serum urate lowering effects when used in combination with XO inhibitors, and with $100 \%$ of patients achieving levels below $4 \mathrm{mg} / \mathrm{dL}$ when combined with allopurinol. The use of AR882 in combination with $\mathrm{XO}$ inhibitors may provide an option for preventing flares as well as tophi reduction in advanced patients.

Disclosure of Interests: Zancong Shen Employee of: arthrosi therapeutics, Chris Colton Employee of: Arthrosi therapeutics Inc, Rongzi Yan Employee of Arthrosi therapeutics Inc, Elizabeth Polvent Employee of: Arthrosi therapeutics Inc, Vijay Hingorani Consultant of: Arthrosi therapeutics Inc, Shunqi Yan Employee of: Arthrosi therapeutics Inc, Li-Tain Yeh Employee of: Arthrosi therapeutics Inc.

DOI: 10.1136/annrheumdis-2021-eular.1215

\section{POS1129 \\ OPTIMIZATION OF DUAL ENERGY COMPUTED TOMOGRAPHY POST-PROCESSING TO REDUCE LOWER LIMB ARTIFACTS IN GOUT}

B. Dubief ${ }^{1}$, J. Avril ${ }^{2}$, T. Pascart ${ }^{3}$, M. Schmitt ${ }^{1}$, R. Loffroy ${ }^{2}$, J. F. Maillefert ${ }^{1}$, P. Ornetti ${ }^{1}$, A. Ramon ${ }^{1}{ }^{1}$ Dijon University Hospital, Rheumatology, Dijon, France; ${ }^{2}$ Dijon University Hospital, Diagnostic and Therapeutic Radiology Department, Dijon, France; ${ }^{3}$ Lille Catholic Hospital, Rheumatology, Lomme, France

Background: Dual energy computed tomography (DECT) is highly accurate for the diagnosis of gout. However, many artifacts have been described $(1,2)$ such as nail bed, skin, beam hardening, sub-millimeter and vascular artifacts). Their presence can lead to a risk of over-diagnosis (false positives)

Objectives: Main objective of this case-control study was to determine the optimal DECT settings post-treatment parameters (ratio and attenuation coefficient $(\mathrm{HU})$ ) in order to reduce the frequency of lower limb artifacts in patients with suspected gout of the lower limbs.

Methods: Seventy-seven patients hospitalized for suspected gout arthritis (feet/ ankles and/or knees) who received a DECT imaging were consecutively included (final diagnosis of 43 gout and 34 other rheumatic disorders). Different post-treatment settings were evaluated from the Syngovia software: an R1 (standard) setting with a ratio at 1.36 and minimum attenuation at $150 \mathrm{HU}$; an R2 setting with a ratio at 1.28 and minimum attenuation at $170 \mathrm{HU}$ and an $\mathrm{R} 3$ setting with a ratio at 1.28 and minimum attenuation at $120 \mathrm{HU}$. The frequency of each artifact according to the 3 settings was determined. Diagnostic accuracy of R1 and R2 settings has been calculated. Correlations between artefacts and patient's clinical characteristics were obtained by performing a Spearman test.

Results: The R2 setting (170 HU, ratio=1.28) significantly reduced the presence of knee and foot/ankle artifacts compared to the standard R1 setting ( $85 \%$ and $94 \%$ decrease in beam hardening and clumpy artifacts in the ankle and foot, respectively ( $p<0.001)$; a decrease of $71 \%, 60 \%$ and $88 \%$ respectively of meniscal beam hardening, beam hardening and submillimeter artifacts in the knee $(p<0.001)$. The use of R3 setting lead to a significant increase of some artifacts (clumpy artifacts, skin artifacts, beam hardening and nail beds.). In addition, our results found a positive correlation between the presence of deposits of knee menisci beam hardening chondrocalcinosis. Body mass index was 